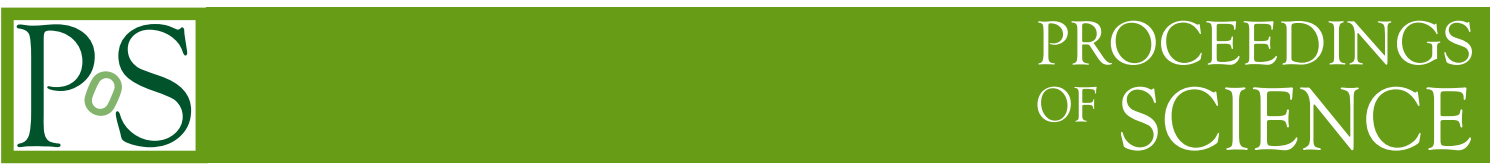

\title{
Where are the large compact-symmetric objects?
}

\section{Pedro Augusto}

Grupo de Astronomia, Universidade da Madeira, Caminho da Penteada, 9000-390 Funchal, Portugal

E-mail: augusto@uma.pt

It is believed that virtually all galaxies went through a phase of activity most predominantly at $z \sim 1$-2. Such 'active galaxies' have the engine at their centres ('active galactic nuclei': AGN). The most favoured explanation is that, somehow (possibly via galaxy merging), a supermassive black hole $(\mathrm{SBH})$ with $10^{6}-10^{9} \mathrm{M}_{\odot}$ forms at the centre of normal galaxies and starts feeding on the surrounding material at the same time spitting out, in two opposite jets of plasma, a fraction of it. Several phenomena are associated with AGN: quasars, blazars, Seyferts and radio galaxies. Radio galaxies, in particular, really 'show off' well how far (i.e. how energetic) the opposite jets can travel (up to a few Mpc). Traditionally, however, only 'mature' radio galaxies ( $10^{9} \mathrm{yrs}$ old) have been catalogued and considered. Hence, there is (still) a mystery as regards their formation mechanisms, which extends to the other types of AGN. In recent years, 'baby' $\left(<10^{3} \mathrm{yrs}\right.$ ) and 'young' $\left(10^{3}-10^{5} \mathrm{yrs}\right)$ radio galaxies have been found and classified, although their numbers are still small (tens). Also, they have many different names, depending on the type of survey and scientific context in which they were found: compact steep spectrum sources (CSS), giga-Hertz peaked spectrum sources (GPS) and compact-medium symmetric objects (C-MSO). The latter have the radio galaxy structure more obvious and correspond to the 'babies' (CSOs; $<1 \mathrm{kpc}$ ) and 'young' (MSOs; 1-15 kpc) radio galaxies. We have established a sample of 37 confirmed CSOs (Augusto et al. 2006). The typical CSO resides on a $z<0.5$ galaxy, has a flat radio spectrum $\left(\alpha_{\text {thin }}<0.5 ; \mathrm{S}_{\nu} \propto \nu^{-\alpha}\right)$, is $<0.3 \mathrm{kpc}$ in size, has an arm length ratio $\leq 2$, and well-aligned $\left(\theta \leq 20^{\circ}\right)$ opposite lobes with a flux density ratio $\leq 10$. The log-size distribution of CSOs shows a sharp drop at $0.3 \mathrm{kpc}$, with only four cases known on $0.3-1 \mathrm{kpc}$. This trend continues, since there are only five flat-spectrum MSOs known (on the full 1-15 kpc size range). In order to find out if this lack of large CSOs and flat-spectrum MSOs is due to poor sampling (lack of surveys that probe efficiently the $0.3-15 \mathrm{kpc}$ size range) or has physical meaning (e.g. if the lobes of CSOs expand as they grow and age, they might become CSSs, 'disappearing' from our statistics), we have built a sample of 157 flat-spectrum radio sources resolved with the VLA-A at $8.4 \mathrm{GHz}$ that were selected from a parent sample of 1743 sources (Augusto et al. 2006). We are now using new, archived and published data to produce and inspect hundreds of multi-frequency multi-instrument maps and models for as many of the 157 sources as possible, in order to finally classify them. Our aim is to substantially increase the number of known CSO-MSOs in the 0.3-15 kpc size range.

From Planets to Dark Energy: the Modern Radio Universe October 1-5 2007

The University of Manchester, $U K$ 
Morphologically, it has been assumed that a compact-medium symmetric object (CSO-MSO) resembles classical Fanaroff \& Riley ([3]) type II radio galaxies (FRIIs), albeit up to $10^{6}$ times smaller $(<15 \mathrm{kpc})$. Operationally, the divide between CSOs and MSOs is at a size of $1 \mathrm{kpc}$ smaller sources are CSOs while larger sources are MSOs. However, one-third of all known CSOs have cores brighter than both lobes ([2]) so they are more like type I radio galaxies (FRIs), in this respect. CSOs are young radio sources $\left(<10^{3} \mathrm{yrs}\right)$. Evidence amounts from kinematics of hotspot motions in ten cases which give ages on $\sim 300-2000$ yrs (e.g. [2]). As regards MSOs, it has not yet been possible to measure kinematics for any; however, other techniques (such as spectral ageing and their sizes) hint at $<10^{5}$ yrs.

The main interest on both of these objects is statistical. If they are:

1. abundant, the favoured evolutionary scheme is: CSO $\rightarrow$ flat-spectrum MSO $\rightarrow$ FRI/FRII; this is called non-self similar evolution (e.g. [4]) since in this case the lobes of CSOs will not expand until a very late stage: after the MSO phase, possibly when $10^{7}-10^{8}$ yrs old, much closer to the FRI/FRII $10^{9}-10^{10}$ yrs ages;

2. not abundant, the favoured evolution is: CSO $\rightarrow$ CSS $\rightarrow$ FRI/FRII; this evolution is selfsimilar, with the lobes expanding as the source grows.

Finding flat-spectrum CSO/MSOs has not been easy. This is likely because nobody has been looking for them. To start with, they have spectra on the wrong side (flat), not very popular for the production of many famous (large and well studied) radio surveys. In addition, they are not the most abundant type of radio source either (perhaps only about one-quarter of all radio sources have a 'thin' emission flat-spectrum). Finally, the few dedicated searches for CSOs have focused on VLBI-sizes $(<0.1 \mathrm{kpc})$, likely missing the population of larger CSOs (if there is one). In order to fix this, and based on previous work ([1]) made on the large Jodrell-VLA Astrometric Survey (JVAS) and Cosmic Lens All-Sky Survey (CLASS) flat-spectrum source surveys (VLA-A at $8.4 \mathrm{GHz}$ ), we started by establishing a parent sample of 1743 sources that have $\mathrm{S}_{8.4}>100 \mathrm{mJy}$, $\alpha_{1.40}^{4.85}<0.5\left(\mathrm{~S}_{\nu} \propto \nu^{-\alpha}\right)$, and $\left|b^{I I}\right|>10^{\circ}$. Then, using a resolution criterion alone, we have defined a sample of 157 sources ([2]): they all have a more than $25 \%$ decrease in visibility from the shortest to the longest baselines on the VLA-A. This criterion is sensitive to components as little as $\sim 0.1$ " apart having a $\sim 7: 1$ flux density ratio. At $z>0.2$ this corresponds to a size of $0.3 \mathrm{kpc}$. It is among these 157 sources that we expect to find many large CSOs and flat-spectrum MSOs. In Figure 1 we present a sum up of the current status in the morphological radio classification of the 157-source sample.

\section{References}

[1] P. Augusto, P.N. Wilkinson, and I.W.A. Browne (1998), Flat-spectrum radio sources with kpc-scale structure, MNRAS 2991159

[2] P. Augusto, J.I. Gonzalez-Serrano, I. Perez-Fournon, P.N. Wilkinson (2006), Flat-spectrum symmetric objects with $\sim 1$ kpc sizes - I. The candidates, MNRAS 3681411

[3] B.L. Fanaroff, J.M. Riley (1974), The morphology of extragalactic radio sources of high and low luminosity, MNRAS 167 P31 


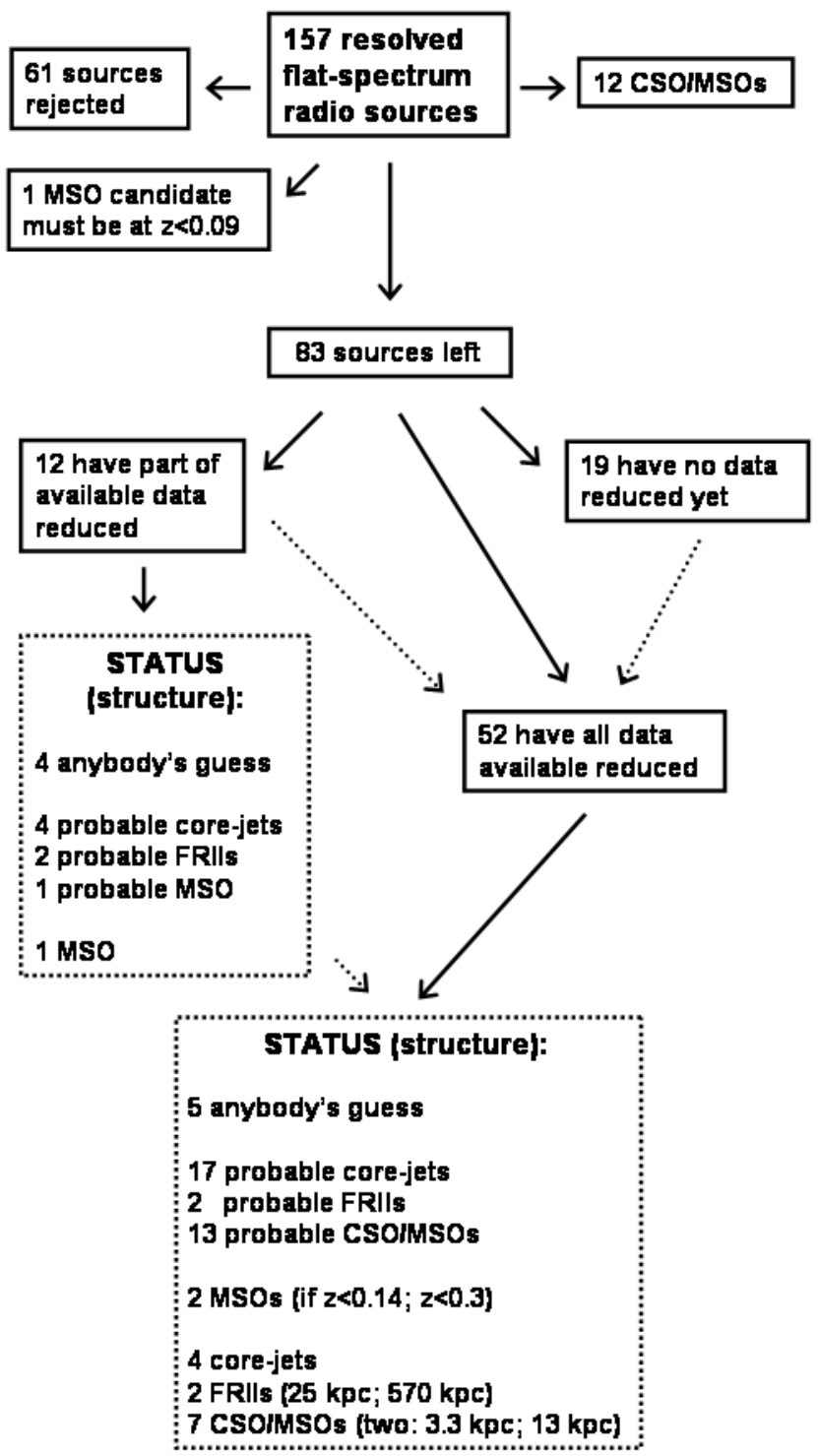

Figure 1: The status of the analysis of the 157-source sample. Dashed arrows indicate future steps. Classification: anybody's guess means that detailed component modeling must be made before learning more about the source structure. Two of the seven confirmed CSO/MSOs (bottom line in the diagram) have redshift information, hence sizes.

[4] W. Tschager, R.T. Schilizzi, H.J.A. Röttgering, I.A.G. Snellen, G.K. Miley (2000), The GHz-peaked spectrum radio galaxy 2021+614: detection of slow motion in a compact symmetric object, $A \& A \mathbf{3 6 0}$ 887 